\title{
Femoral Mycotic Aneurysm Secondary to Community: Acquired Methicillin: Resistant Staphylococcus aureus Infection
}

\author{
Fahad Fayyaz Butt ${ }^{\mathrm{a}}$ Mohamed Ashraf Kandath ${ }^{\mathrm{a}}$ Fatima Farid Mir ${ }^{\mathrm{a}}$ \\ Thouqeer Ahmed ${ }^{b}$ \\ aPediatrics Department, Dubai Hospital, Dubai, United Arab Emirates; ${ }^{\text {b}}$ Radiology Department, Dubai Hospital, \\ Dubai, United Arab Emirates
}

\section{Keywords}

Methicillin-resistant Staphylococcus aureus · Abscess ·

Femoral artery aneurysm

\begin{abstract}
We present the case of a thirteen-year-old, morbidly obese boy admitted with right hip septic arthritis and severe sepsis. Blood and joint aspirate cultures confirmed growth of methicillinresistant Staphylococcus aureus. Parenteral antibiotic therapy was administered according to culture sensitivity reports, and several joint re-explorations were required for drainage of pus recollection at the hip site. Over a month-long inpatient stay culminated in an episode of profuse fresh bleeding from the right hip wound site, with subsequent $C T$ angiography confirming mycotic aneurysm development at the deep branch of the femoral artery. Emergent endovascular embolization and coil insertion ensued, allowing steady improvement till discharge home.

(c) 2021 The Author(s)

Published by S. Karger AG, Basel
\end{abstract}

\section{Introduction}

Methicillin-resistant Staphylococcus aureus, an organism once linked near exclusively to hospital-acquired infections, has been increasingly observed to cause disease among the general community. The body of evidence on methicillin-resistant Staphylococcus aureus infections is ever expanding across all patient populations and clinical settings. Noteworthy is the broad spectrum of disease, ranging from localized skin infections to cerebral abscesses, necrotizing pneumonias, and mycotic aneurysms.

A mycotic aneurysm is described as an infectious intrusion in the wall of an artery with the development of a blind saccular outpouching which is adjacent and in contact with the arterial lumen [1]. Infective aneurysms can evolve secondary to bacteremia and sepsis, ending in spontaneous arterial rupture and death if treatment was delayed. Hence, it is associated with significant morbidity and mortality. Our report of femoral artery mycotic aneurysm following septic methicillin-resistant Staphylococcus aureus arthritis aims to further raise attention toward the close relation between these 2 entities.

\section{Case Report}

A thirteen-year-old boy with a known case of morbid obesity, hyperglycemia on metformin with renal dysfunction referred from a tertiary hospital as a case of septic arthritis on IV antibiotics with complaints of fever, right hip and left leg pain, and limping. Six days prior, he had trauma when he was playing volleyball; he hit himself and fell to the ground. The pain started and progressively karger@karger.com www.karger.com/dmj

Karger $\stackrel{\text { ' }}{=}$

BOPEN ACCESS
(C) 2021 The Author(s)

Published by S. Karger AG, Basel

This article is licensed under the Creative Commons AttributionNonCommercial-NoDerivatives 4.0 International License (CC BYNC-ND) (http://www.karger.com/Services/OpenAccessLicense). Usage and distribution for commercial purposes as well as any distribution of modified material requires written permission.
Correspondence to:

Fatima Farid Mir, fatimafmir@gmail.com 
increased since then. He did not complain of a history of fever, rash, abdominal pain, or vomiting.

In the ER, the child had a temperature of $102.2 \mathrm{~F}\left(39.1^{\circ} \mathrm{C}\right)$, heart rate 119 beats/min, blood pressure $113 / 56 \mathrm{~mm} \mathrm{Hg}$, and capillary refill $<2 \mathrm{~s}$. He was tachypneic and distressed requiring oxygen supplementation. Joint examination revealed tenderness over the right groin, painful restricted movement of the right hip, no active movement, and the patient kept the right hip in external rotation. The left ankle and leg showed redness, warmth, and tenderness, with intact distal neurovascular state of both lower limbs. Initial investigations were remarkable showing a white cell count of $29.0 \times$ $10^{3} / \mu \mathrm{L}$, neutrophils $83 \%$, band neutrophils, $12 \%$, lymphocytes $1 \%$, hemoglobin $9.5 \mathrm{~g} / \mathrm{dL}$, platelets $128 \times 10^{3} / \mu \mathrm{L}$, C-reactive protein $290 \mathrm{mg} / \mathrm{L}$, procalcitonin $5.87 \mathrm{ng} / \mathrm{mL}$, and erythrocyte sedimentation rate $90 \mathrm{~mm} / \mathrm{h}$.

The child was admitted to the PICU as a case of severe sepsis with right hip septic arthritis, started on IV meropenem and IV vancomycin. Initial blood cultures, right hip joint fluid aspirate, and biopsy culture revealed MRSA growth. Antibiotics were adjusted according to sensitivity, and biochemical and clinical response, and he completed a course of IV teicoplanin, clindamycin, and rifampicin. A USS Doppler was done, and there was no evidence of thrombosis. DVT prophylaxis was given. A USS hip showed moderate right hip effusion. MRI showed features of myositis involving the iliopsoas, obturator, and the gluteus muscles and osteomyelitis involving the right upper femur, right iliac, and pubis bones. Perfusion and lactate remained normal on serial monitoring.

Right hip arthrotomy was done at presentation and 2 times afterward in a span of 2 weeks due to repeated pus collections. Fever recurred on day 30; complete septic workup was done including fluid and wound culture growing Pseudomonas aeruginosa, which responded to IV meropenem course.

On the 25th day of admission, he developed recurrent frank oozing of blood from the wound site that was amenable to pressure with no source identified on exploration. On the 40th day, he underwent a CT angiogram in view of recurrent continuous bleeding from the exploration wound site, which revealed outpouching arising from the deep muscular branch of the right common femoral artery, and an urgent embolization was done with coil insertion (Fig. 1, 2).

Follow-up blood and joint fluid aspirate cultures showed no growth. Distal neurovascular status remained intact. No residual end-organ injury. Outpatient follow-ups in the subsequent months revealed mild pain, residual limitation of right hip movement, and patient preference toward wheelchair ambulation. Serial hip Xrays sadly showed development of right-sided slipped capital femoral epiphysis with steady progression toward avascular necrosis of the femoral head (Fig. 3). He was lost to follow-up thereafter.

\section{Discussion}

Community-acquired methicillin-resistant Staphylococcus aureus is a major public health problem primarily related to health care but no longer confined to intensive care units, acute care hospitals, or any healthcare institution [2]. Methicillin-resistant Staphylococcus au-

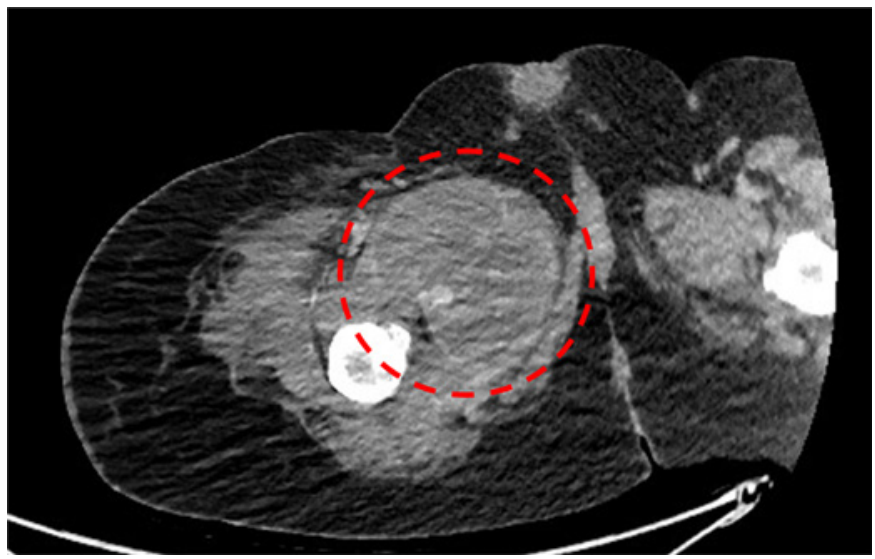

Fig. 1. CT scan: the medial and posterior compartments of the thigh are enlarged with heterogeneous enhancement, showing multiple pockets of collections varying in sizes likely representing post-infective/inflammatory collection.

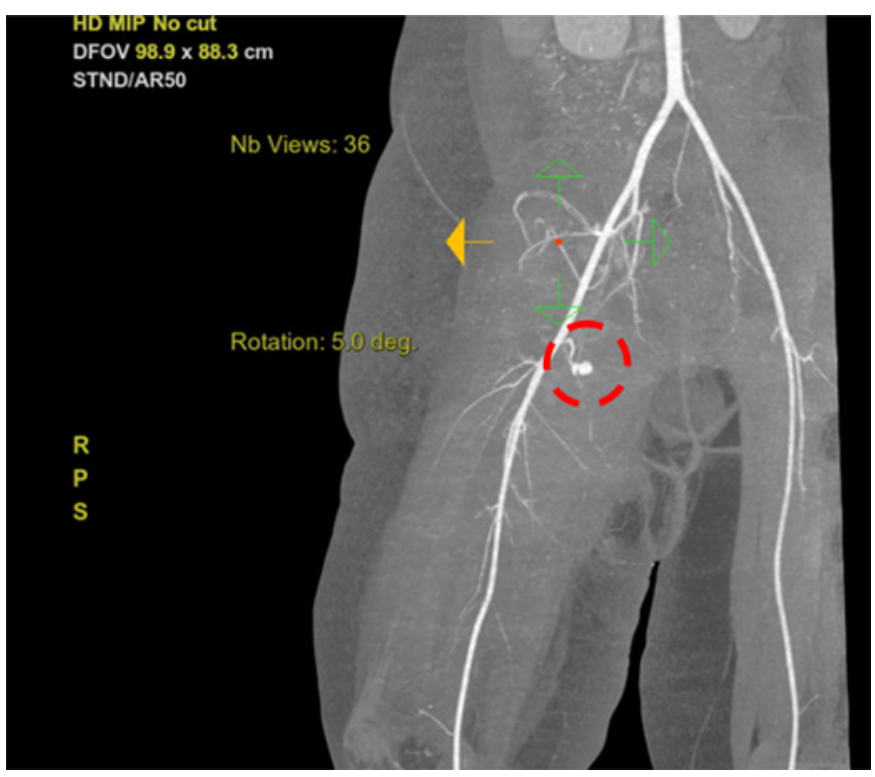

Fig. 2. CT angiography: a lobulated contrast-filled outpouching measuring $16 \times 10 \mathrm{~mm}$ seen to be arising from the deep muscular branch of the right common femoral artery in medial aspect of the proximal thigh, likely representing a mycotic pseudoaneurysm. 


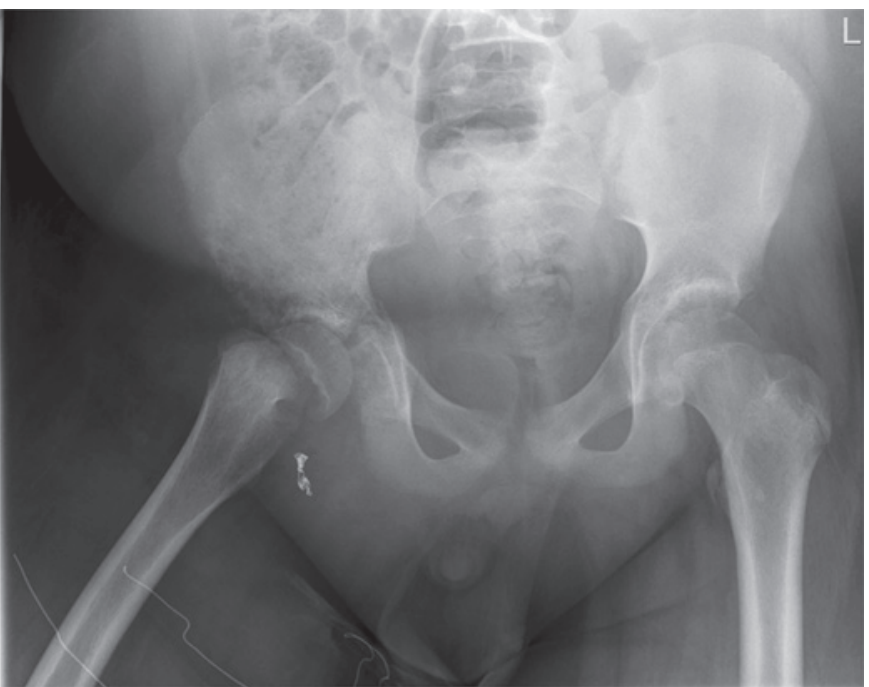

Fig. 3. Right hip X-ray: severe right-sided slipped capital femoral epiphysis.

veloping diffuse osteomyelitis, refractory septic arthritis, and fragile mycotic aneurysmal dilation of local arteries, despite aggressive medical therapy. Several authors have described the development of a mycotic aneurysm over a pre-existing structural defect, for example, ductus arteriosus aneurysm [7] or post-cardiac surgery [8]. It is worth noting however that aneurysmal degeneration of the arterial wall can also be a result of infection due to bacteremia or septic embolization [5].

Mycotic aneurysms are a rare but potentially lifethreatening condition. Numerous recommendations exist for the management strategies based upon the location of the affected artery, degree of involvement, and most importantly patients' underlying cardiovascular reserve for intervention.

Broadly, the conventional strategy for treatment is via a multidisciplinary approach targeting prompt and aggressive antibiotic therapy, along with surgical intervention as deemed appropriate in the patient's individual circumstance [9]. Severe sepsis followed by persistent elevation of inflammatory markers and poor general condition led our vascular surgery and interventional radiology teams to opt for an endovascular embolization and coil insertion route, rather than open surgery for resection and graft placement. Our patient's postintervention outcome proved to be positive in the short run, but the unfortunate progression toward avascular necrosis of the femoral head seems attributable to the culminated effect of morbid obesity, severe infection, and aneurysm formation.

\section{Conclusion}

Methicillin-resistant Staphylococcus aureus infections are capable of causing significant end-organ damage, both locally and systemically. Our patient has demonstrated a rare complication of femoral mycotic aneurysmal dilation secondary to severe localized abscess formation, requiring endovascular coil insertion. The aforementioned intervention managed to control acute symptoms; however, over the course of the year, the child has progressed to avascular necrosis of the femoral head with impaired independent mobilization.

\section{Acknowledgements}

The authors would like to acknowledge S.S. for her contribution to the Introduction section.

\section{Statement of Ethics}

Guardians have provided their informed consent to publish their child's case.

\section{Conflict of Interest Statement}

The authors have no conflicts of interest to declare.

\section{Funding Sources}

The authors did not receive any funding.

\section{Author Contributions}

F.F.B. and F.F.M. collected data, wrote manuscript, and collated and reviewed the literature. M.A.K. closely reviewed, guided, and edited the final paper. T.A. analyzed and elaborated upon radiologic images.

\section{References}

1 Lee WK, Mossop PJ, Little AF, Fitt GJ, Vrazas JI, Hoang JK, et al. Infected (Mycotic) aneurysms: spectrum of imaging appearances and management. Radiographics. 2008 Nov 1; 28(7):1853-68.

2 Nadle J, Klevens RM, Morrison MA. Invasive methicillin-resistant staphylococcus aureus infections in the United States. JAMA. 2007 Oct 17;298(15):1763-71 
3 Stauffer WM, Siwek AD, Kamat D, KemplerMeyer E. Visual diagnosis: a 14-year-old male who has fever and rash. Pediatr Rev. 2003 Dec 1;24(12):424-9.

4 Williams MT, Jiang H. Diffuse cerebral petechial hemorrhage in an 8-year-old girl with MRSA pneumonia and sepsis. Neurology. 2014 Jan 21;82(3):282.

5 Naidu DP, Quinones JA, Lutschg KW, Balaguru D. Healthy adolescent with a mycotic aortic aneurysm from community-acquired methicillin-resistant staphylococcus aureus. Tex Heart Inst J. 2017 Aug;44(4):279-82.
6 Khandanpour N, Chaudhuri A, Roebuck DJ, Armon MP. Neonatal mycotic internal iliac aneurysm due to methicillin-resistant staphylococcus aureus (MRSA) septicaemia successfully treated by coil embolisation. Eur J Vasc Endovasc Surg. 2007;33(6):687-9.

7 Stewart A, Dyamenahalli U, Greenberg SB, Drummond-Webb J. Ductus arteriosus aneurysm with community-acquired methicillin-resistant staphylococcus aureus infection and spontaneous rupture: a potentially fatal quandary. Pediatrics. 2006 Jun 1; 117(6):e1259-62.
8 Atiyah M, Mohsin S, Al Faraidi L, Al-Hawri K, Al Otay A, Al Najashi K. Surgical management of ascending aortic pseudoaneurysm in a 2-year-old boy: a case report. J Med Case Rep. 2018 Apr 17;12(1):98.

9 Lee NY, Kan CD, Chi CY, Chen PL, Lee HC, Chang CM, et al. Community-acquired methicillin-resistant staphylococcus aureus mycotic aneurysm. Intern Med. 2010;49(6):623-5. 